\title{
Article
}

\section{Deep Removal of Humic Compounds by Scavengers Combination}

\author{
Tetiana MITCHENKO, Nataliya MAKAROVA, Andrey MITCHENKO, Helena SHEVCHUK \\ Chemical Department, National Technical University of Ukraine, 37, Pr.Pobedy, Kiev, 03056, Ukraine \\ Gudrun ABBT-BRAUN \\ University of Karlsruhe, Engler-Bunte-Institute, Engler-Bunte-Ring 1, D-76131 Karlsruhe, Germany
}

(Manuscript submitted February 19, 2003; accepted April 15, 2003)

\begin{abstract}
The humic compounds removal from tap water by separate scavengers and their combinations was investigated. It was shown, that adsorption of humic compounds can be improved using a combination of weak-base macroporous anion-exchange resin Dowex MWA-1 and bituminous granular activated carbon F-300 in comparison with the individual performance of these adsorbents. Utilization of the investigated combination of adsorbents for water purification allows to increase velocities of filtration (decrease the dimension of adsorption plants), increase capacity of activated carbon and decrease demand of the regeneration agent. Purified water has a low content of humic compounds and a constant mineral composition.
\end{abstract}

\section{Research and results}

The high concentration of humic compounds (HC) in tap water of the majority Ukrainian cities causes necessity for development of new effective methods for water treatment up to the normative requirements for the drinking water.

The water purification from $\mathrm{HC}$ is carried out traditionally by activated carbons (AC) of various natures. The efficiency of this process essentially depends on activated carbon porous structure. The researches of HC adsorption from water by activated carbons with the known physico-chemical and structural characteristics [1] were conducted for a choice of AC possessed maximal sorption ability to HC presented in tap water. The experiments have shown investigated carbons under the capacities characteristics can be arranged as the following order: wood $<$ coconut $<$ anthracite $<$ bituminous.

F300 has shown the best sorption properties regarding to $\mathrm{HC}$ in a number investigated AC. This adsorbent represents the reagglomerated bituminous crushed activated carbon with the high content of both micro- and mesopores, formed by channels between smallest carbon particles. Such AC granules structure formed in reagglomeration process. The size and specific volume of $\mathrm{AC}$ pores defines its high adsorption ability to $\mathrm{HC}$. As was shown earlier the HC sorption occurs mainly in pores with the size 2-20 nm. [2]

The results of experiments (Fig. 1) have shown, that the AC F300 is the most effective for removal of small fractions of humic compounds with molecular weight less than 1000 Dalton. It is explained by its porous structure. In spite of the fact that the share of this HC fraction in Dnieper water is rather insignificant (less than 20\%),[3] such property of F300 is highly useful, since the HC fraction with molecular weight less than 1000 Dalton 
is most toxic and requires especially deep removal for the treatment of drinking water and water for beverage and food production.

The opportunity to use activated carbon of such type for removal of small HC fractions, water dechlorination and deodoration simultaneously makes this adsorbent especially perspective for deep sorption purification of the drinking water and food-industry water in the combined technologies.

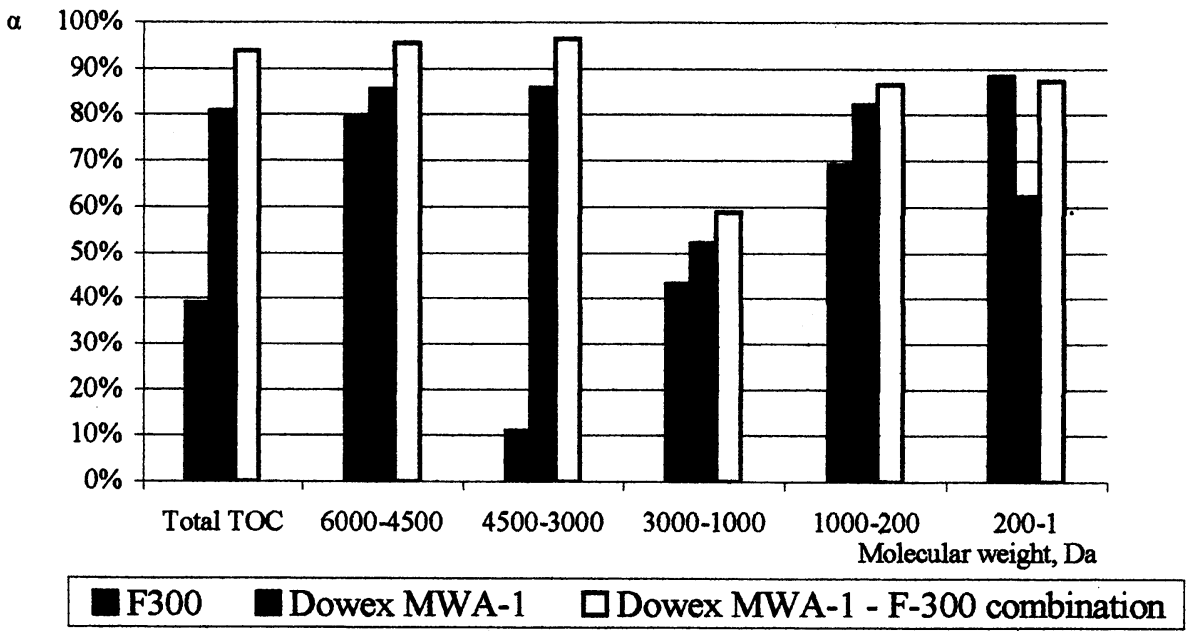

Fig.1. Uptake ( $\alpha$ ) of HC fractions by F300, Dowex MWA-1 and combination MWA-1-F300.

At the same time data about kinetic features of $\mathrm{HC}$ sorption by activated carbons, submitted on a Fig. 2, have shown, that the efficiency of $\mathrm{HC}$ removal (especially for large fractions) from water by activated carbon F-300 essentially depends on volumetric velocity of solution, passed through the adsorbent. When the $\mathrm{HC}$ concentration in tap water is high (permanganate oxidability up to $7 \mathrm{mgO}_{2} / \mathrm{dm}^{3}$ ) the $\mathrm{AC}$ is efficient for the $\mathrm{HC}$ removal from water only at the volumetric velocity no more than 10 bed volume per hour (BV/h). Hereupon the large-sized devices and large loadings $\mathrm{AC}$ at the water treatment are required and both, capital and operational expenses increase. Besides, the impossibility of the effective regeneration of activated carbon results in the frequent replacement of carbon in apparatuses.

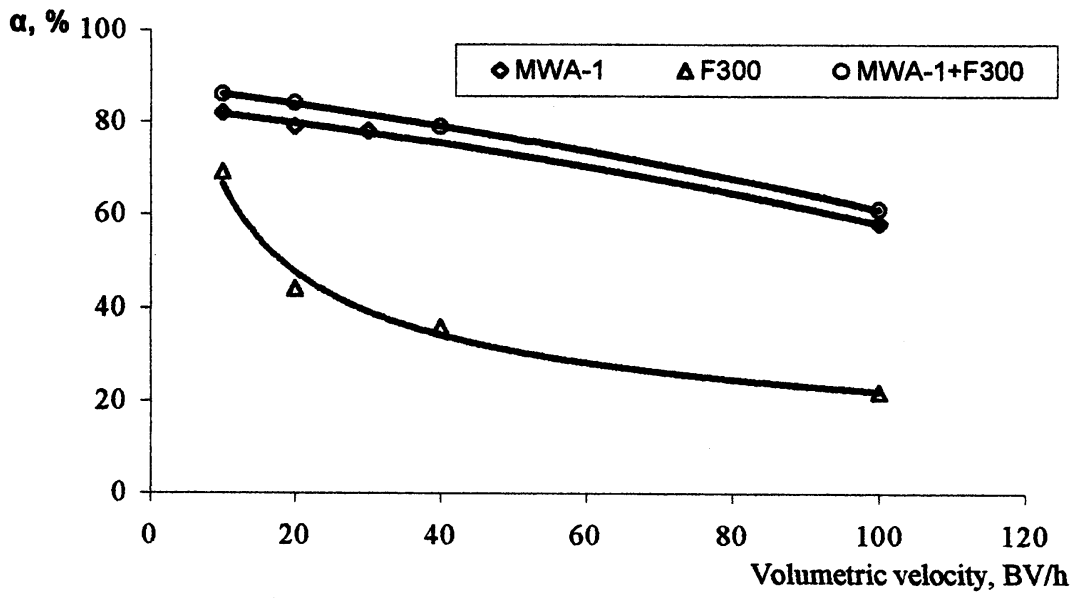

Fig.2. The impact of the solution volumetric velocity on the HC uptake $(\alpha)$.

The anion exchange resins - scavengers use to exclude the specified lacks. As the results of experiments have shown, the application of weak base anion exchange resin Dowex MWA-1 allows to realize water purification 
from $\mathrm{HC}$ at enough high velocities, without changing its mineral contents. However uptake of all HC fraction by this anion resin does not exceed $80 \%$, and for small $\mathrm{HC}$ fractions - $60 \%$ (Fig. 1). Besides this adsorbent is not intended for adsorption of chlorine and chlororganic derivatives.

It was logical to assume, that in this case the adsorbents combination (including weak base anion resin and activated carbon) will allow to raise efficiency of water purification from $\mathrm{HC}$, chlorine and chlorine derivatives simultaneously.

We conducted dynamic experiments with Kiev tap water to purify from both $\mathrm{HC}$ and active chlorine by a combination of weak base macroporous anion exchange resin Dowex MWA-1 and bituminous activated carbon F-300. The permanganate oxidability of initial tap water changed within the limits $3.7-5.1 \mathrm{mgO} / \mathrm{dm}^{3}$ and chlorine contents was $0.3-0.5 \mathrm{mg} / \mathrm{dm}^{3}$. Water was passed consistently through glass columns filled Dowex MWA-1 and F-300 at the volumetric velocity $40 \mathrm{BV} / \mathrm{h}$. The anion-exchange resin was regenerated periodically by the $10 \%$ $\mathrm{NaCl}$.

As the results of this experiment were shown (Table 1, Fig. 3), the use of weak-base anion exchange resin Dowex MWA-1 and bituminous activated carbon F300 as combination for the tap water purification allows to reach the higher level of $\mathrm{HC}$ removal in comparison with individual adsorbents. Herewith the residual free chlorine in the purified water was absent.

Table 1 The efficiency of $\mathrm{HC}$ adsorption by anion-exchange resin Dowex MWA-1, activated carbon F300 and the combination of two.

\begin{tabular}{|c|c|c|c|c|c|c|c|}
\hline \multirow{2}{*}{\multicolumn{2}{|c|}{ Adsorbents }} & \multicolumn{2}{|c|}{$\begin{array}{c}\mathrm{HC} \text { contents, } \\
\mathrm{mgO}_{2} / \mathrm{dm}^{3}\end{array}$} & \multirow{2}{*}{$\begin{array}{c}\text { Volumetric } \\
\text { velocity, } \\
\text { BV/h }\end{array}$} & \multirow{2}{*}{$\begin{array}{c}\text { Contact } \\
\text { time, } \\
\text { min. }\end{array}$} & \multirow{2}{*}{$\begin{array}{c}\mathrm{HC} \\
\text { uptake, } \\
\%\end{array}$} & \multirow{2}{*}{$\begin{array}{c}\text { Equilibrium } \\
\text { dynamic volume } \\
\text { capacity, } \\
\mathrm{mgO}_{2} / \mathrm{cm}^{3} \\
\end{array}$} \\
\hline & & $\begin{array}{c}\text { Tap } \\
\text { water }\end{array}$ & $\begin{array}{c}\text { Purified } \\
\text { water }\end{array}$ & & & & \\
\hline \multirow{2}{*}{\multicolumn{2}{|c|}{ MWA-1 }} & 8.7 & 2 & 20 & 3 & 77 & \\
\hline & & 8.7 & 2.1 & 40 & 1.5 & 76 & \\
\hline \multirow[t]{5}{*}{ F300 } & individual & 4.5 & 1.4 & 10 & 6 & 69 & 5.5 \\
\hline & individual & 4.5 & 2.9 & 40 & 1.5 & 36 & 2.0 \\
\hline & after MWA-1 & 2 & 0.9 & 10 & 6 & 55 & 7.5 \\
\hline & after MWA-1 & 2 & 1.1 & 20 & 3 & 44 & 6.8 \\
\hline & after MWA-1 & 2 & 1.28 & 40 & 1.5 & 36 & 5.1 \\
\hline \multicolumn{2}{|c|}{ Combination } & 8.7 & 1.1 & 20 & 3 & 87 & \\
\hline \multicolumn{2}{|c|}{ MWA-1-F300 } & 8.7 & 1.28 & 40 & 1.5 & 85 & \\
\hline
\end{tabular}

It is also important that influence of the kinetic aspects upon adsorption efficiency decreases and HC capacity increases when F-300 uptakes HC from water previously treated by Dowex-MWA-1. The shift in molecular weigh distribution of $\mathrm{HC}$ in water after passing through this resin explains this phenomenon.

Thus, despite the decrease of $\mathrm{HC}$ concentration in treated water the activated carbon F-300 showed the higher capacity and better kinetic characteristics. This can happen due to elimination of shielding of the active sites of $\mathrm{AC}$ by large-size molecules of $\mathrm{HC}$, which were adsorbed by anion resin.

These reasons cause synergetic increase in the efficiency of $\mathrm{HC}$ adsorption by the combination of two adsorbents in comparison with their individual performance. 
The specified combination of adsorbents allows to reduce oxidability of tap water for most of Ukrainian cities less than up to $2 \mathrm{mgO}_{2} / \mathrm{dm}^{3}$ at volumetric velocity up to $40 \mathrm{BV} / \mathrm{h}$ and also to remove chlorine and its derivatives from water.

The realization of such combined method for the tap water purification from $\mathrm{HC}$ allows to achieve essential decrease of capital expenses (in comparison with traditional technologies) due to use of the smaller size sorption devices.

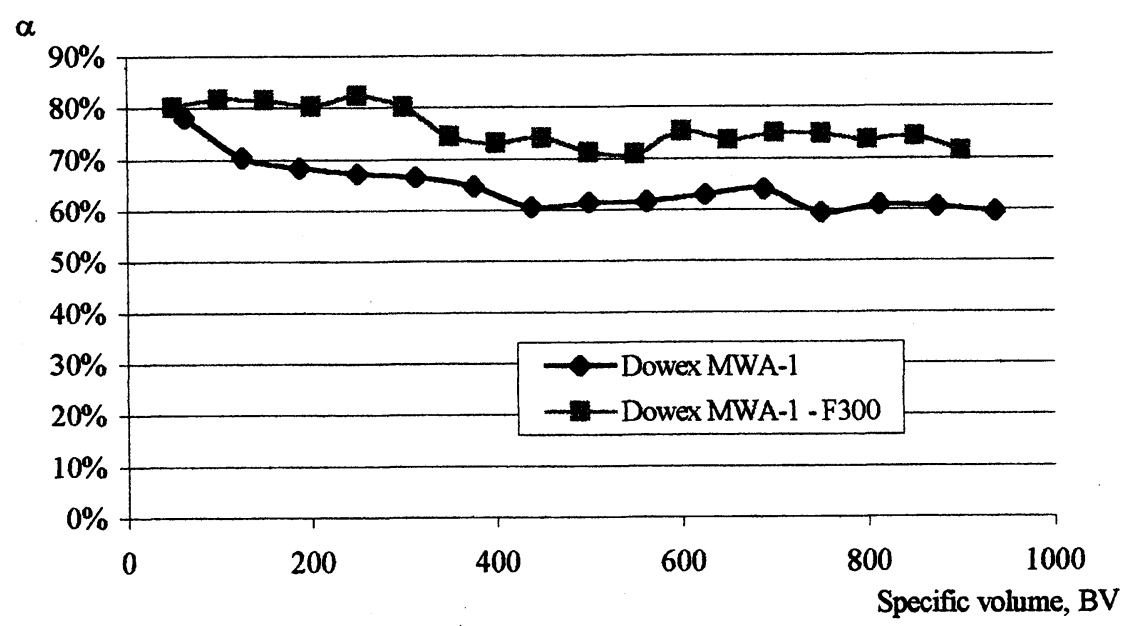

Fig. 3. HC uptake from Kiev tap water by anion-exchange resin Dowex MWA-1 and by composition Dowex MWA-1 - F-300 at the volumetric velocity $40 \mathrm{BV} / \mathrm{h}$.

The researches have shown, the investigated combination of adsorbents Dowex MWA-1 and F-300 allows not only to reach a high uptake for both, $\mathrm{HC}$ and chlorine from the tap water at the high volumetric velocity of water, passed through the adsorbents layer, but also to prolong essentially the service life of AC. As the pilot test have shown, after the preliminary treatment of tap water by weak-base anion exchange resin the service resource F300 before replacement achieves $15-20000 \mathrm{BV}$ at the $40 \mathrm{BV} / \mathrm{h}$, and the interval between regenerations for Dowex MWA-1 makes 1000 BV. Achievement of similar water quality by F300 without preliminary water passing through Dowex MWA-1 is possible only at the volumetric velocity no more than $10 \mathrm{BV} / \mathrm{h}$. Herewith carbon resource makes no more than 2.5-3 $000 \mathrm{BV} / \mathrm{h}$. Dowex MWA-1, used for water purification without the following treatment by $\mathrm{F} 300$, reduces $\mathrm{HC}$ uptake to the same, but its regeneration are necessary much more often (through 200-300 BV) and, besides water will demand in addition dechlorination and deodoration.

Thus, utilization of the investigated combination of adsorbents for the industrial water purification from $\mathrm{HC}$ can allow essentially improve both, quality of clearing, and economic characteristics of process.

\section{Acknowledgement}

The investigations were sponsored by the INTAS ("Water Purification for Food Production, INTAS 00-174).

\section{References}

1) T.Y. Mitchenko, N.T. Kartel, A.A. Mitchenko, H.A. Shevchuk. Proceedings of the Intern. Conf. "Carbon 99", USA, 700-701 (1999).

2) A. Mitchenko. Proceedings of the Conference on membranes in drinking and industrial water production, Paris, 2, 581-586 (2000).

3) Mitchenko A., Frimmel F., Gremm T., MitchenkoT, Makarova N. Interfaces against pollution, 2-nd Intern. Conf., Hungary, 140 (2002). 